This item was submitted to Loughborough's Research Repository by the author.

Items in Figshare are protected by copyright, with all rights reserved, unless otherwise indicated.

\title{
Solvent flux through dense polymeric nanofiltration membranes
}

PLEASE CITE THE PUBLISHED VERSION

PUBLISHER

(C) Elsevier

VERSION

AM (Accepted Manuscript)

LICENCE

CC BY-NC-ND 4.0

REPOSITORY RECORD

Robinson, J.P., E.S. Tarleton, C.R. Millington, and Arian Nijmeijer. 2009. "Solvent Flux Through Dense Polymeric Nanofiltration Membranes”. figshare. https://hdl.handle.net/2134/4787. 
This item was submitted to Loughborough's Institutional Repository (https://dspace.lboro.ac.uk/) by the author and is made available under the following Creative Commons Licence conditions.

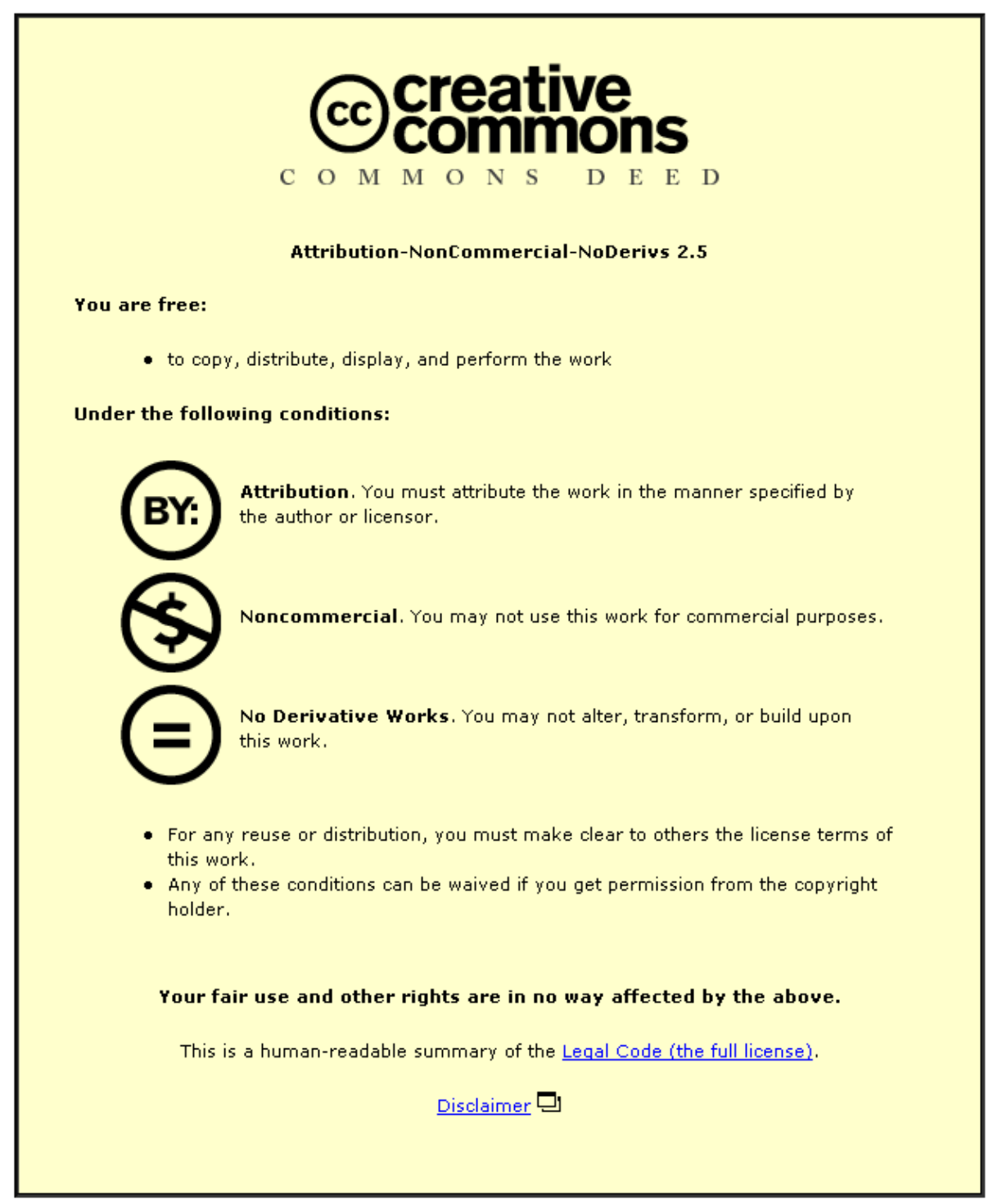

For the full text of this licence, please go to: http://creativecommons.org/licenses/by-nc-nd/2.5/ 


\title{
SOLVENT FLUX THROUGH DENSE POLYMERIC NANOFILTRATION MEMBRANES
}

\author{
J.P. Robinson ${ }^{a}$, E.S. Tarleton ${ }^{a}$ (e.s.tarleton@lboro.ac.uk), C.R. Millington ${ }^{\mathrm{b}}$ and A. Nijmeijer ${ }^{\mathrm{c}}$ \\ ${ }^{a} A d v a n c e d$ Separation Technologies Group, Department of Chemical Engineering, Loughborough \\ University, Loughborough, Leicestershire LE11 3TU, UK. \\ bShell Global Solutions, Cheshire Innovation Park, P.O. Box 1, Chester CH1 3SH, UK. \\ ${ }^{c}$ Shell Global Solutions International BV, P.O. Box 38000, 1030 BN Amsterdam, The Netherlands.
}

\begin{abstract}
This work examines the flux performance of organic solvents through a polydimethylsiloxane (PDMS) composite membrane. A selection of $n$-alkanes, i-alkanes and cyclic compounds were studied in deadend permeation experiments at pressures up to $900 \mathrm{kPa}$ to give fluxes for pure solvents and mixtures between 10 and $100 \mathrm{I} \mathrm{m}^{-2} \mathrm{~h}^{-1}$. Results for the chosen alkanes and aromatics, and subsequent modelling using the Hagen-Poiseuille equation, suggest that solvent transport through PDMS can be successfully interpreted via a predominantly hydraulic mechanism. It is suggested that the mechanism has a greater influence at higher pressures and the modus operandi is supported by the non-separation of binary solvent mixtures and a dependency on viscosity and membrane thickness. The effects of swelling that follow solvent-membrane interactions show that the relative magnitudes of the Hildebrand solubility parameter for the active membrane layer and the solvent(s) are a good indicator of permeation level. Solvents constituting a group (e.g. all n-alkanes) induced similar flux behaviours when corrections were made for viscosity and affected comparable swelling properties in the PDMS membrane layer.
\end{abstract}

\section{KEYWORDS}

Nanofiltration; Membrane; PDMS; Organic solvents

\section{INTRODUCTION}

Transport mechanisms and process limitations are relatively well understood for aqueous nanofiltration (NF) systems, and the majority of research has been performed in this area. A body of work has also been assembled on the use of membranes for the removal of suspended matter from organic solvents. However, the separation of organic solute compounds from organic solvents using membrane technology has been addressed by very few workers, and little is known of the fundamental transport and removal mechanisms. As a precursor this paper reports data for the transport of solvents and mixtures through polydimethylsiloxane (PDMS) membranes, the former being a representative simulant for the feed to a new industrial process.

PDMS membranes are dense materials used in pervaporation, vapour permeation and gas separation processes. Recently, a number of workers have evaluated PDMS and other dense membranes for use in non-aqueous nanofiltration applications such as homogeneous catalyst recovery and the de-acidification of vegetable oils. Researchers have studied and previously attempted to model the behaviour of organic solvents and polymeric membranes. Initial development of thermodynamic theories was carried out by Paul and Ebra-Lima [1] as early as 1970 whereas studies of polymer-solvent interactions were documented by Flory [2] in the 1950s, and other workers since $[3,4]$. Two distinct processes have been reported for different membranes and operating regimes. The transport processes for high pressure liquid systems in all areas from Microfiltration (MF) to Reverse Osmosis (RO) are pressure-driven physical mechanisms. Systems utilising dense membranes, such as pervaporation, gas separation and vapour permeation are considered to be governed by chemical transport processes such as adsorption and diffusion. 


\section{Physical Transport}

According to Darcy's Law, physical or hydraulic transport through membranes and other media with physical pores is pressure-driven. For liquids, the flux behaviour can be described by the Hagen-Poiseuille equation for viscous flow:

$$
J=\left(\frac{\varepsilon r^{2}}{8 L T}\right)\left(\frac{\Delta P}{\mu}\right)
$$

where $J$ is the solvent flux, $\varepsilon$ the porosity, $r$ the average pore radius, $\Delta P$ the differential pressure across the membrane, $\mu$ the liquid viscosity, $L$ the membrane thickness and $\tau$ the tortuosity factor. Pore tortuosity, $T$, is normally defined as the ratio of the true length of the flow path and the straight-line distance between the beginning and end points [5]. In many cases the pore geometry and geometry distribution are unknown, so the tortuosity factor reduces to an adjustable parameter.

For NF/RO membranes and aqueous systems the osmotic pressure, $\Pi$, is commonly used as a parameter in transport equations. The water flux in reverse osmosis can be described by $J \approx(\Delta P-\Delta \Pi)$, however there is some debate as to whether osmotic pressure is most applicable to non-aqueous systems. A preferential sorption-capillary flow model, as described by Kimura and Sourirajan [6], can be used to describe the transport mechanism in RO. The model assumes that solvent and solute transport takes place in pores, and that the solvent is preferentially adsorbed onto the pore walls, with solute rejection taking place at the membrane surface. For non-aqueous systems, possible interactions between the solvent and polymeric membranes have lead to the development of alternative transport equations. Machado et al. [7] proposed a resistance-in-series model to describe the flux of organic solvents through composite polymeric membranes. Three significant resistances to mass transport were identified as viscous flow in the membrane top layer, viscous flow in the porous support and hydrophilic/hydrophobic resistances. The resulting equation for solvent flux is:

$$
J=\frac{\Delta P}{\varphi\left[\left(\Delta \gamma+f_{1} \mu\right)+f_{2} \mu\right]}
$$

where $\varphi$ is a single parameter incorporating the membrane characteristics (porosity, tortuosity, thickness), $\Delta y$ is the surface energy difference between the membrane and solvent and $f_{1}$ and $f_{2}$ are constants incorporating the individual mass transfer coefficients and pore radii. The model predicts that hydrophobicity plays an important role in solvent flux; polar solvents (those with a high surface tension) are expected to have a low flux through hydrophobic membranes, and a high flux through hydrophilic membranes. Zwijnenberg et al. [8] also report the importance of the surface energy difference in a study of polar and non-polar solvents with hydrophilic membranes.

Permeation through the membrane pores is only possible when the difference in surface energy can be overcome by the applied pressure. Bhanushali et al. [9] have shown that solvent surface tension is inversely proportional to flux for hydrophobic membranes, as polarity of organic solvents is strongly related to surface tension.

\section{Chemical Transport}

In pervaporation, gas separation and vapour permeation with dense membranes, chemical transport mechanisms are considered predominant. The favoured concept is the solution-diffusion model first proposed by Lonsdale et al. [10], where transport occurs by a substance dissolving in the membrane and diffusing through it. The separation potential of the membrane is therefore determined by differences in solubility and diffusivity (see Wijmans and Baker [11]). 
Many workers have studied the sorption and diffusion behaviour of solvents with PDMS membranes, with both sorption and diffusion being the principal rate-determining step(s) depending on the solvent-polymer system. Sorption of solvents in polymers is non-ideal $[12,13]$, i.e. Henry's Law does not apply. The 'Hildebrand solubility parameter', $\delta$, is one method of estimating the affinity of a solvent for a particular polymer [14]. The parameter takes into account hydrogen-bonding, polar and dispersive effects, and can be assigned to both solvents and polymers from their molecular structures and chemical groups. Solvents and polymers with a similar value of Hildebrand parameter would be expected to interact strongly, whilst those with dissimilar values would not. Such an approach has been verified by Bhanushali et al. [9], in a study of the sorption behaviour of a range of solvents in PDMS.

An alternative approach to non-ideal solubility is the Flory-Huggins theory, which relates the activity of a penetrant inside a polymer to the degree of swelling and a semi-adjustable interaction parameter. Flory-Huggins theory predicts sorption behaviour of many organic solvents into PDMSlike membranes [15], although polar solvents such as alcohols require a more sophisticated approach [16]. The diffusion of penetrant molecules through polymer networks can be ideal (Fickian) or non-ideal (non-Fickian). Substances with a low solubility in a membrane will generally exhibit Fickian diffusion, whereas high concentrations of penetrant will yield non-ideal behaviour [17]. Whether a membrane is in a glassy or rubbery state can also influence the diffusive behaviour of a penetrant, with the 'free-volume' of rubbery polymers being an important factor [18].

In conclusion, literature suggests that the transport mechanisms for PDMS are dependent on the system operational parameters rather than the material itself. Gas separation, pervaporation and vapour permeation processes utilising PDMS membranes are generally well described by the solution-diffusion model, whereas the same membranes used for high-pressure solvent permeation processes can be modelled with both Darcy's Law and the Solution-Diffusion model.

\section{EXPERIMENTAL}

\section{Materials}

The membranes used in the current study were radiation crosslinked PDMS - Polyacrylonitrile (PAN) composites supplied by GKSS Forschungszentrum. The nominal PDMS thickness was 2 $\mu \mathrm{m}$, and an $\mathrm{O}_{2} / \mathrm{N}_{2}$ selectivity of 2.2 was reported for the membranes [19]. When received, the $\mathrm{N}_{2}$ permeance was checked and found to be $280 \pm 10$ barrer $\left(2.13 \times 10^{-8} \mathrm{~m}^{2} \mathrm{~s}^{-1} \mathrm{kPa}^{-1}\right)$, assuming the nominal $2 \mu \mathrm{m}$ thickness to be representative. $\mathrm{O}_{2} / \mathrm{N}_{2}$ selectivity and pure nitrogen permeation data confirmed the selective layer in the membrane to be PDMS [20].

PDMS thickness was verified by SEM images of the membrane cross-section as shown in Figure 1. In order for the true cross-section to be viewed, the membrane was freeze-fractured to eliminate artefacts of the cutting blade. A number of SEM images showed the thickness to be largely consistent at $2 \mu \mathrm{m}$, but variations between 1.5 and $3 \mu \mathrm{m}$ were noted in some places.

The solvents used in the study were n-hexane, n-heptane, cyclohexane and xylene (mixture of isomers) as supplied by Sigma-Aldrich. Methanol and ethanol were obtained from Fisher Scientific and isomeric alkanes were supplied by Shell Global Solutions. These compounds comprise representative simulants of those found in the industrial process to which the study relates. The compositions of the solvents were determined by gas-chromatography, and their densities and viscosities calculated from pure component data at $20^{\circ} \mathrm{C}$. The isomeric alkanes comprised ihexane (98\% 3-methylpentane), i-heptane (95\% 3-methylhexane) and i-octane (99\% 2,2,4trimethylpentane). The viscosity of a mixture $(\mu \mathrm{m})$ was calculated using a well established expression for hydrocarbon liquids [21]: 
$\mu_{m}=\left(\sum_{1}^{n} x_{i} \mu_{i}^{1 / 3}\right)^{3}$

where $\mu_{i}$ is the individual component viscosity and $x_{i}$ the mole fraction.

\section{Test Apparatus}

Solvent flux was measured using the membrane module shown schematically in Figure 2 . The module comprised two stainless steel discs of $150 \mathrm{~mm}$ diameter and $20 \mathrm{~mm}$ thickness. The bottom plate was milled such that a $75 \mathrm{~mm}$ diameter sintered plate fitted flush with the top surface. The flat-sheet membrane was cut into a disc to overlap the sintered plate by $10 \mathrm{~mm}$. A $3 \mathrm{~mm}$ thick PTFE gasket was placed over the top to clamp the membrane in position, facilitate a hydraulic seal and provide space between the membrane and top plate to be filled with liquid. Inlet/outlet channels on the top plate allowed the module to operate in both deadend and crossflow modes*.

The solvents were supplied to the module with the apparatus shown in Figure 3. Compressed nitrogen was used to pressurise the reservoir and force the solvent through a dip-tube out of the reservoir to the membrane module. Pressures were measured via a gauge mounted in the solvent reservoir that was calibrated at frequent intervals using a commercial Druck DPI 603 calibration apparatus. As all experiments reported in this paper were carried out in deadend mode, the pressure in the reservoir corresponded to that above the membrane surface.

\section{Experimental Method}

Before commencing a permeation experiment, the valve on the membrane module outlet was opened fully, and a small pressure applied to the fluid in the reservoir to bleed excess air from the system. $100 \mathrm{ml}$ of solvent was then run through the module to remove remaining gas and to flush away any residual solvent from the previous test. The module exit valve was then closed, and the pressure increased to the test value between 10 and $900 \mathrm{kPa}$; the lower pressure ranges, although outside those normally used in NF, were investigated to provide more relevant information as the study relates to a process operating within the stated pressure range. The permeate was left to drain for 10 minutes to establish a steady-state before being collected in a narrow-necked flask. Experiments were given enough time to allow approximately $100 \mathrm{ml}$ of solvent to permeate the membrane. Permeation rate was measured by weighing the collected permeate after a specified time.

When transferring between different solvents, $500 \mathrm{ml}$ of the new solvent was run through the reservoir and the module to flush away unwanted residue.

\section{Repeatability}

Three samples of PDMS membrane were used to obtain the results reported in this paper. nheptane was employed as a 'standard' solvent to establish a datum baseline where (for fixed operating conditions) the flux between different membranes varied by $\pm 10 \%$ whereas measurements of flux through individual membranes varied by $\pm 2 \%$ over periods of use spanning several days. Whether a membrane was stored in a swollen-state or allowed to dry had no noticeable impact on flux performance and no special handling was required to maintain the permeation/separation capability of the original membrane. No appreciable degradation in membrane performance was noted for the range of solvents tested.

To account for the different membranes and associated variability, the flux-pressure relationship for n-heptane was initially determined. n-heptane fluxes were also measured at 300, 600 and $900 \mathrm{kPa}$

\footnotetext{
* The crossflow mode has been used in solute rejection work that is reported elsewhere [28].
} 
before the flux-pressure relationship of a new solvent was recorded. The ratio of solvent flux : nheptane flux was calculated in each series of experiments, and that ratio used to calculate the solvent flux based on the original $n$-heptane data. This re-calculation enabled solvent fluxes to be accurately compared.

\section{RESULTS AND DISCUSSION}

The data reported here are representative, other similar data have been acquired to confirm those shown.

\section{Pure Solvents}

The flux of alkanes and aromatic solvents ranged from $10-100 \mathrm{I} \mathrm{m}^{-2} \mathrm{~h}^{-1}$ depending on the solvent and pressure used. Methanol and ethanol, however, had much lower fluxes due to unfavourable interactions with the membrane that could not be accurately quantified because the evaporation rate from the permeate collection beaker was significant compared to the flux. No further attempt was made to measure alcohol fluxes, instead it was estimated that pure alcohol flux is around two orders of magnitude lower than that of alkanes.

Figure 4 shows typical flux measurements at pressures of $300-900 \mathrm{kPa}$ for $\mathrm{n}$-hexane and cyclohexane. Least squares regression of the data yielded linear relationships over the tested pressure range. In all cases, however, the regressed line for n-alkane, i-alkane and cyclic solvents failed to pass through the origin on the flux-pressure plot and always exhibited a positive intercept on the $y$-axis between 1.1 and $3.2 \mathrm{I} \mathrm{m}^{-2} \mathrm{~h}^{-1}$. These values do not necessarily represent a physical effect, i.e. a measurable flux at zero pressure, rather they are indicative of non-linear behaviour over the available pressure range up to $900 \mathrm{kPa}$. Table 1 shows gradients and intercepts of the regressed flux-pressure relationships for all tested solvents in the 300-900 kPa pressure range.

To evaluate flow resistance of the PAN substrate alone, samples were obtained from GKSS and permeation experiments performed with $\mathrm{n}$-heptane and xylene gave fluxes of 3.85 and $2.38 \mathrm{I} \mathrm{m}^{-2} \mathrm{~h}^{-}$ ${ }^{1} \mathrm{kPa}^{-1}$ respectively. These are approximately two orders of magnitude higher than corresponding measurements with the composite membrane (see Table 1) and the substrate appears to have a negligible impact on overall flux performance. Linear regression of the flux-pressure data for the PAN layer did not yield a positive $y$-axis intercept as was the case for the PDMS-PAN composite. The result suggests that solvent transport through the PAN occurs via a single mechanism and that any structural changes in the layer have a negligible effect on flux levels. This in turn infers that the behaviour observed in Figure 4 (and others) is due exclusively to the presence of the PDMS layer.

Further investigations were carried out with xylene and n-heptane at lower pressures. The apparatus in Figure 3 was modified by the addition of a second regulator and a $0-250 \mathrm{kPa}$ pressure gauge that enabled determinations of flux behaviour at pressures as low as $10 \mathrm{kPa}$. The results for xylene are presented in Figure 5, where a deviation from the 300-900 kPa trend is apparent. Figure 6 shows corresponding low and high pressure data for $n$-heptane where there is much less distinction between the two regions. From the complete data set obtained with all solvents a transition in the flux-pressure relationship was apparent at pressures in the region of $300-350 \mathrm{kPa}$. Although the perceived differences in gradient could be small and perhaps insignificant, for instance on Figure 6 , regression analysis showed that two distinct relationships invariably offered a better correlation than a single line. In Figure 5, for example, a regression using all the data yields a correlation coefficient, $R^{2}=0.9786$. It is noted in passing that for an experiment performed with no applied nitrogen pressure and just a $10 \mathrm{~cm}$ head of solvent, corresponding to a pressure of $0.7 \mathrm{kPa}$, no readily detectable solvent flux was obtained. 
To the knowledge of the authors similar data to those shown in Figures 4-6 have not been explicitly highlighted by other workers, although some appear to have overlooked or dismissed similar results. Although Bhanushali et al. [9] report a zero intercept for various solvents and a PDMS membrane at pressures from $500-5000 \mathrm{kPa}$, several of their datasets can be reinterpreted in a manner similar to that shown on Figure 5. Gibbins et al. [21] report that steady-state methanol permeations through a STARMEM 122 membrane obey Darcy's law at pressures up to $6000 \mathrm{kPa}$. A significant number or their results don't support this conclusion as correlations to the fluxpressure data can be readily interpreted to have a non-zero intercept of between 20 and $40 \mathrm{I} \mathrm{m}^{-2} \mathrm{~h}$ 1. Scarpello et al. [22] studied solvent flux through a range of organic nanofiltration membranes at pressures of 1000-4000 kPa and reported a zero intercept. In a further experiment by the authors, a sample of the membrane with an identical PAN substrate and a $10 \mu \mathrm{m}$ thick PDMS layer was obtained from GKSS. Although the different thickness will inevitably lead to some variation in the extent of radiation induced crosslinking, the linear regressed gradient and intercept of the fluxpressure relationship with $n$-heptane were reduced to approximately one fifth of those obtained for the membrane with the $2 \mu \mathrm{m}$ PDMS layer.

There are several potential explanations for the results shown in Figures 4-6. Experimental error was eliminated as no discrepancy in the experimental technique or fault in the testing apparatus could be identified, and many repeated experiments, as well as other data, confirm the results shown in this paper. One possible consideration is membrane compaction. Although pressures in the present study were limited to $900 \mathrm{kPa}$, it seems unlikely that a step change in membrane structure would occur at pressures of 300-350 kPa and then no further compaction occur (as evidenced by the linearity of the flux-pressure graphs from 300-900 kPa). Another potential explanation is that two distinct mechanisms are in operation, namely hydraulic and chemical transport. Since PDMS membranes have been found to allow sorption and diffusion of organic solvents in pervaporation and vapour permeation [23, 24], it is reasonable to assume that these diffusive processes can occur in nanofiltration. In some studies with PDMS membranes workers have found that flux is a function of solvent viscosity $[7,9]$, which may imply a form of hydraulic transport mechanism. Contrary to this, others researchers have found that chemical transport via the solution-diffusion mechanism is prevalent $[1,25]$. It is possible that a combination of hydraulic and chemical solvent transport exists with PDMS membranes.

The data presented in Figures 4-6 (and later in Figures 7, 9 and 10) are not intended to promote or degrade a particular theory, however, in the opinion of the authors the reported data suggest that a PDMS membrane can behave, and be modelled, as if it had physical pores. To be consistent with this interpretation, and thus obey the Hagen-Poiseuille model [9], from Equation (1) a plot of flux against $(\Delta P / \mu)$ would be expected to yield a straight line with gradient $\left(\varepsilon r^{2} / 8 L \tau\right)$. Figure 7 shows

such a plot for all tested solvents and Table 2 reports relevant densities and viscosities. It is evident that three distinct correlations exist. n-alkanes and i-alkanes show different but consistent gradients, whereas the two cyclic compounds lie on the same regression line, albeit with a higher gradient than that recorded for the n-alkanes ${ }^{* *}$. Cyclohexane, a cyclic alkane, does not obey the same trend as the other alkanes, probably due to the different molecular shape. The data indicate that apparently similar solvents affect their own membrane properties, that is their own specific value of $\left(\varepsilon r^{2} / 8 L T\right)$. This is a somewhat surprising result as PDMS is a dense membrane with no defined porous structure, yet 'solvent groups' can be interpreted to behave as if the membrane has pores, which in turn infers a hydraulic solvent transport mechanism.

\footnotetext{
${ }^{* *}$ In terms of actual solvent flux, the measured values for the cyclic alkanes were always lower than the fluxes recorded for $\mathrm{i}$ - and $\mathrm{n}$-alkanes at equivalent pressures. On Figure 7 the viscosity correction (on the x-axis) results in the datapoints for the cyclic alkanes lying above the other data. Measured fluxes for the i-alkanes were between those of the cyclic and n-alkanes. These data correspond to the relative magnitudes of the Hildebrand solubility parameters for the PDMS membrane layer and the specific solvent.
} 
The specific gradients observed for each solvent group are a consequence of the unique swelling effects of the different solvents. As noted, the relative magnitudes of the Hildebrand solubility parameter $(\delta)$ are indicative of the swelling propensity of PDMS, and the relationship between the gradient from the Hagen-Poiseuille plot, $\left(\varepsilon r^{2} / 8 L \tau\right)$, and $\delta$ is shown in Figure 8. Although solvents with a limited range of $\delta$ are shown, Figure 8 indicates that a solvent with a higher value of $\delta$ generally exhibits a steeper gradient on the Hagen-Poiseuille plot. However, given the low recorded fluxes for methanol $(\delta=29.2)$ and ethanol $(\delta=26.5)$ with the membrane, the plot may be misleading as solvents exhibiting significantly higher $\delta$ will also generate much lower values for $\left(\varepsilon r^{2} / 8 L \tau\right)$ and a maximum is likely to be recorded for an intermediate value of $\delta$.

\section{Solvent Mixtures}

The flux behaviour of two binary, cyclic/straight-chain solvent systems, n-heptane/xylene and nhexane/cyclohexane, was also investigated. In all cases, the measured fluxes of the n-alkanes were higher than those for the cyclic compounds at equivalent pressures (as would be expected from comparisons of $\delta$ values), and no change in the composition of the solvent mixtures was detected following passage through the membrane. These results could be inferred to support a hydraulic transport mechanism as identified in Figure 7. The non-separation of solvent mixtures is in agreement with the data of Paul et al. [26] and Machado et al. [7].

The flux behaviour of the two binary systems was modelled with Equation (1) as shown in Figures 9 and 10. For both solvent systems, intermediate mixtures followed the same trend as for the cyclics (cyclohexane and xylene), with the pure straight-chain alkanes outlying the rest of the data. Since the chemistry of the cyclic compounds is quite different the effect is likely to be influenced by their cyclic shape. Bowen and Welfoot [26], working with aqueous NF systems, have suggested that straight chain molecules are able to become more ordered when permeating through a confined space (such as the transport region within PDMS) and increase their viscosity, particularly close to pore walls. In MF and UF the membrane pore size is much greater than the molecular dimensions of the permeating solvent. However, the same cannot be said of NF, and it is plausible that the transport regions within the membrane are of the same order of size as the solvent molecules. Viscosity increases of $10 \%$ for n-heptane and $30 \%$ for $n$-hexane would be required to produce the deviations shown in Figures 9 and 10. Alternatively, the deviations are more likely to be due to differences in the swelling of the PDMS membrane layer. Given the nominal $2 \mu \mathrm{m}$ thickness of PDMS on the membrane, and the inherent problems of producing much thicker samples with an identical amount of radiation induced crosslinking on which measurements may be possible, the degree of swelling in the PDMS layer is currently difficult to quantify with sufficient accuracy $^{* * *}$. Swelling of PDMS in the presence of solvent is qualitatively well documented and the inference from Figure 10 (in particular) is that the degree of swelling imparted by the $50 \%$ cyclohexane $/ 50 \% \mathrm{n}$-hexane mixture is very similar to that due to the cyclohexane solvent alone. This in turn suggests that the degree of swelling induced by the cyclic/straight-chain mixture is nonlinear with composition. A threshold fraction of the (greater swelling) straight-chain solvent is required to produce similar behaviour to that of the pure solvent. Although not directly comparable, Yoo et al. [4] have demonstrated non-linear swelling behaviour with mixtures of $n$-hexane/acetone and n-hexane/ethanol in PDMS and further data for the binary solvent systems used in this study are currently being acquired by the authors.

\footnotetext{
${ }^{* * *}$ If the Hagen-Poiseuille model correctly predicts the presence of pores in the PDMS layer then it is theoretically possible to calculate an average pore size from, for instance, the gradient of the plots in Figures 7, 9 and 10. However, the porosity and tortuosity are unknown variables and swelling is likely to change the layer thickness (which may also be a function of pressure) and lead to unreliable calculations. Pore size estimates are possible from rejection measurements and data for a 9, 10 Diphenylanthracene solute indicate mean pore sizes between $1.88 \mathrm{~nm}$ and $7.97 \mathrm{~nm}$ depending on the solvent and model used [28].
} 


\section{CONCLUSIONS}

It is suggested that the transport of a range of alkane and aromatic solvents through a PDMS/PAN composite membrane can be successfully interpreted using the Hagen-Poiseuille equation. The PDMS membrane layer behaves as if physical pores are present to an extent dependent on the degree of solvent induced swelling. At pressures above $\sim 300 \mathrm{kPa}$ solvent transport can be considered to occur by a hydraulic mechanism, whereas below this threshold level a second mechanism is also more apparent and may involve a combination of sorption and diffusion. The non-separation of solvent mixtures passing through the membrane and a dependency on viscosity and membrane thickness support a hydraulic mechanism, however, the authors recognise that flux levels predicted by the solution-diffusion model can also account for these parameters to varying degrees. Whilst more work is undoubtedly required to fully justify the hypothesis proposed sufficient data have been obtained to warrant that investigation and hence deepen overall understanding.

\section{ACKNOWLEDGEMENTS}

Shell Global Solutions (UK), are acknowledged for supplying the project funding, technical hardware and isomeric solvents. EPSRC are also acknowledged for part-funding this project. The PDMS membranes used in this study were kindly supplied by GKSS Forschungszentrum.

\section{NOMENCLATURE}

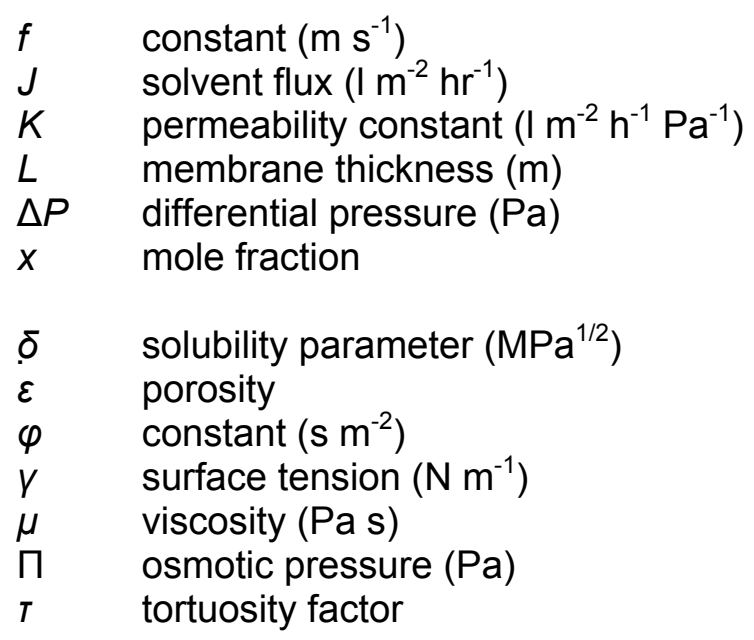

barrer $\times 10^{-10} \mathrm{~cm}^{3}(\mathrm{STP}) \cdot \mathrm{cm} \mathrm{cm} \mathrm{c}^{-1}(\mathrm{~cm} \cdot \mathrm{Hg})^{-1}$

\section{REFERENCES}

1. D.R. Paul and O.M. Ebra-Lima, Pressure-induced diffusion of organic liquids through highly swollen polymer membranes. J. App. Poly. Sci. 14 (1970) 2201-2224.

2. P.J. Flory, Principles of Polymer Chemistry, Cornell University Press 1953.

3. Y-.M. Sun and J. Chen. Sorption/desorption properties of ethanol, toluene and xylene in poly (dimethylsiloxane) membranes. J. App. Polym. Sci. 51 (1994) 1797-1804. 
4. J.S. Yoo, S.J. Kim and J.S. Choi, Swelling equilibria of mixed solvent/poly(dimethylsiloxane) systems. J. Chem. Eng. Data 44 (1999) 16-22.

5. M. Sahimi, Flow and Transport in Porous Media and Fractured Rock. VCH 1995.

6. S. Kimura and S. Sourirajan, Analysis of data in reverse osmosis with porous cellulose acetate membranes. AlChEJ. 13 (1967) 497.

7. D.R. Machado, D. Hasson and R. Semiat, Effect of solvent properties on permeate flow through nanofiltration membranes. Part II: Transport model. J. Membrane. Sci. 166 (2000)6369.

8. H.J. Zwijnenberg, A.M. Krosse, K. Ebert, K-V. Peinemann and F.P. Cuperus, Acetone-stable nanofiltration membranes in deacidifying vegetable oil. J. Am. Oil. Chem. Soc. 76 (1999) 83-87.

9. D. Bhanushali, S. Kloos, C. Kurth and D. Bhattacharyya, Performance of solvent-resistant membranes for non-aqueous systems: solvent permeation results and modelling. $J$. Membrane. Sci. 189 (2001) 1-21.

10. H. Lonsdale, U. Merten and R. Riley. Transport of cellulose acetate osmotic membranes. J. Appl. Polym. Sci. 9 (1965) 1341.

11. J.G. Wijmans and R.W. Baker. The solution-diffusion model: A review. J. Membrane. Sci. 107 (1995) 1-21.

12. Y-M. Sun and J. Chen, Sorption/desorption properties of ethanol, toluene and xylene in poly(dimethylsiloxane) membranes. J. App. Poly. Sci. 51 (1994) 1797-1804.

13. E. Favre, P. Schaetzel, Q.T. Nguygen, R. Clement and J. Neel, Sorption, diffusion and vapour permeation of various penetrants through dense poly(dimethylsiloxane) membranes: A transport analysis. J. Membrane. Sci. 92 (1994) 169-184.

14. A.F.M. Barton, CRC Handbook of Solubility Parameters and Other Cohesion Parameters. CRC Press 1983.

15. E. Favre, Q.T. Nguygen, P. Schaetzel, R. Clement and J. Neel, Sorption of organic solvents into dense silicone membranes. Part 1 - validity and limitations of Flory-Huggins and related theories. J. Chem. Soc. Faraday Trans. 1993 89(24) 4339-4346.

16. E. Favre, R. Clement, Q.T. Nguygen, P. Schaetzel and J. Neel, Sorption of organic solvents into dense silicone membranes. Part 2 - Development of a new approach based on a clustering hypothesis for associated solvents. J. Chem. Soc. Faraday Trans. 1993 89(24) 4347-4353.

17. J.S. Vrentas, J.L. Duda and W.J. Huang, Regions of Fickian diffusion in polymer-solvent systems. Macromol. 19 (1986) 1718-1724.

18. J.L. Duda and J.M. Zielinski, Free-Volume Theory. Chapter 3 in Diffusion in Polymers. P. Neogi (Ed.) Marcel Dekker 1996.

19. GKSS Forschungszentrum. Technical Datasheet. October 2001.

20. M.H.V. Mulder, Basic Principles of Membrane Technology. Kluwer Academic Publishers. 1991. 
21. R.H. Perry and D.W. Green (Eds). Perry's Chemical Engineers Handbook, $7^{\text {th }}$ Edition, 2-367. McGraw-Hill 1998.

22. E. Gibbins, M. D’Antonio, D. Nair, L.S. White, L.M. Freitas dos Santos, I.F.J. Vankelecom and A.G. Livingston. Observations on solvent flux and solute rejection across solvent resistant nanofiltration membranes. Desalination 147 (2002) 307-313.

23. J.T. Scarpello, D. Nair, L.M. Freitas dos Santos, L.S. White and A.G. Livingston. The separation of homogeneous organometallic catalysts using solvent resistant nanofiltration. $J$. Membrane. Sci. 203 (2002) 71-85.

24. H-K Oh, K-H Song, K-R Lee and J-M Rim. Prediction of sorption and flux of solvents through PDMS membrane. Polymer 42 (2001) 6305-6312.

25. C.K. Yeom, S.H. Lee, H.Y. Song and J.M. Lee. Vapor permeations of a series of $\mathrm{VOC} / \mathrm{N}_{2}$ mixtures through PDMS membranes. J. Membrane. Sci. 198 (2002) 129-143.

26. D.R. Paul, J.D. Paciotti and O.M Ebra-Lima. Hydraulic permeation of liquids through swollen polymeric networks. II. Liquid mixtures. J. Appl. Polym. Sci. 19 (1975) 1837-1845.

27. W.R. Bowen and J.S. Welfoot. Modelling the performance of membrane nanofiltration - critical assessment and model development. Chem. Eng. Sci. 57 (2002) 1121-1137.

28. J.P. Robinson, E.S. Tarleton, C.R. Millington and A. Nijmeijer, Evidence for swelling-induced pore structure in dense PDMS nanofiltration membranes. FILTRATION 4(1) 2004 50-56. 
FIGURES AND TABLES



Figure 1 - 20 kV SEM cross-section image showing a $2 \mu \mathrm{m}$ PDMS layer on the PAN substrate.

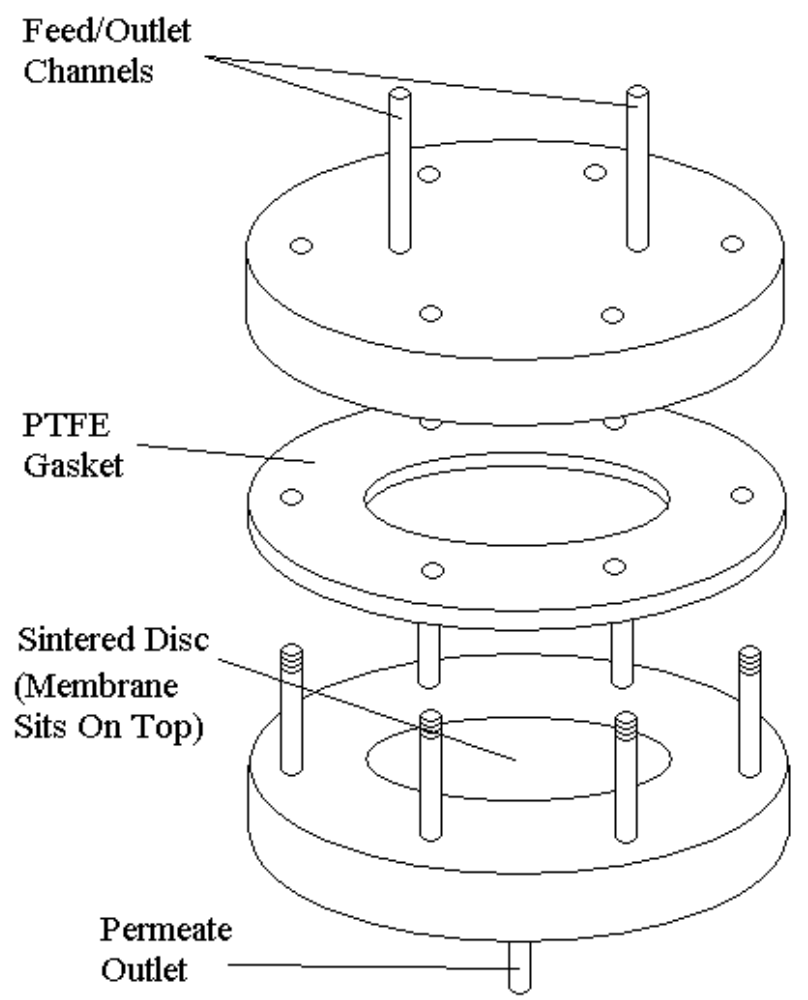

Figure 2 - Schematic of the flat-sheet membrane module. 


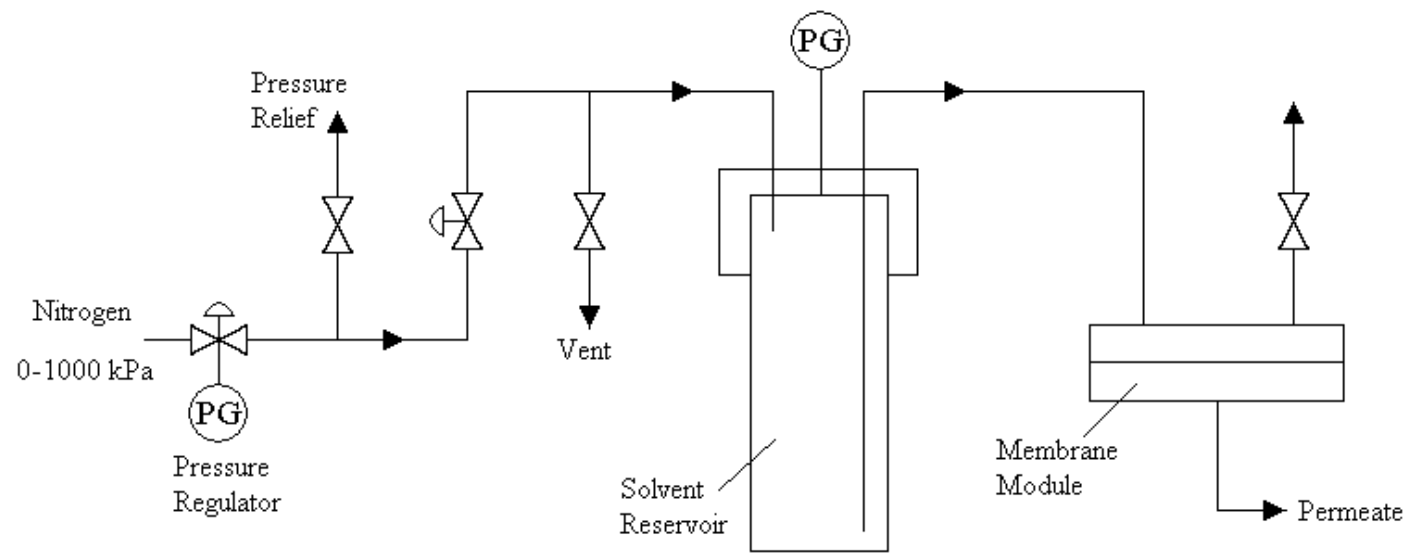

Figure 3 - Schematic of the solvent permeation apparatus.

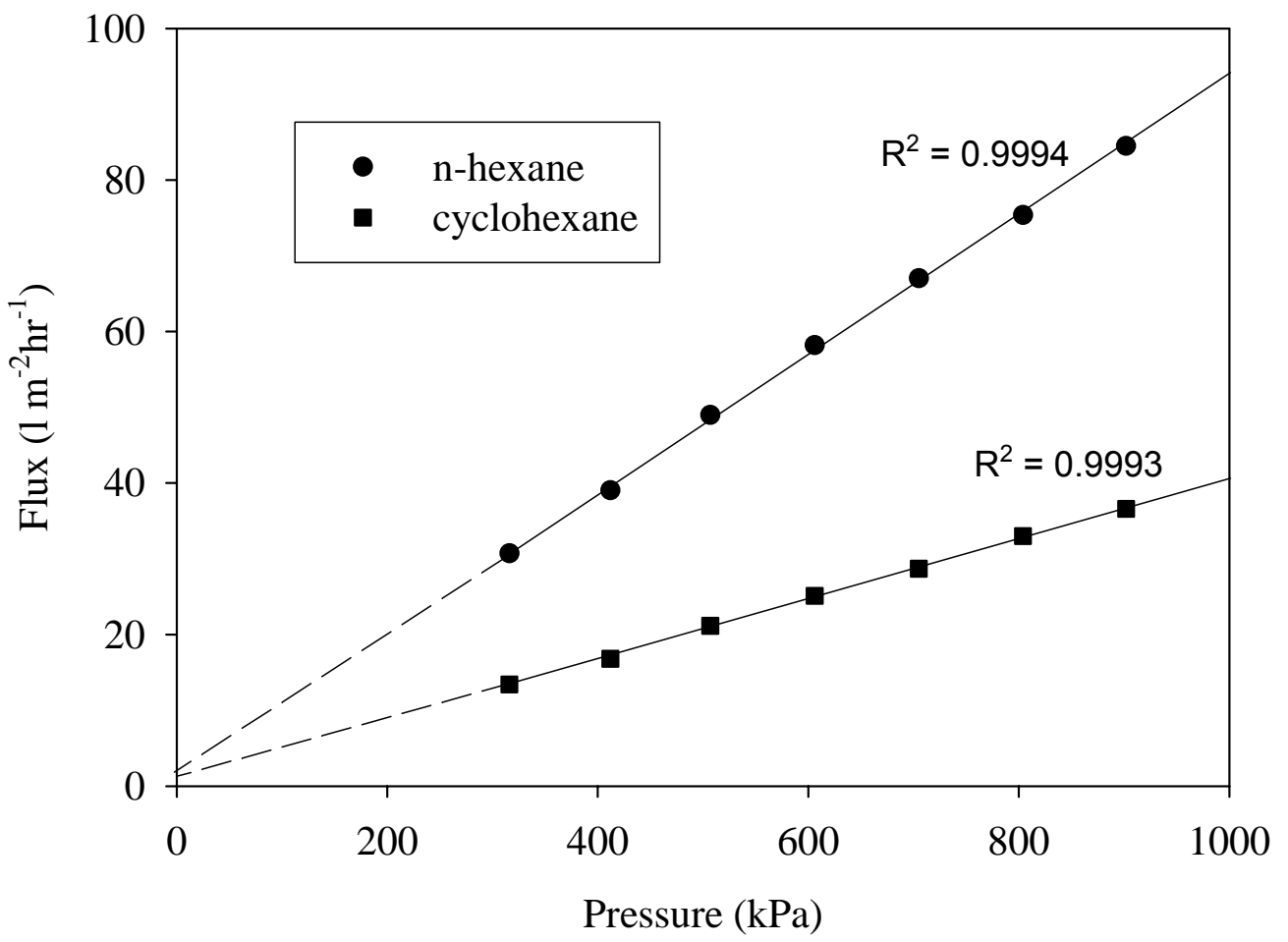

Figure 4 - Flux-pressure relationship for n-hexane and cyclohexane. 


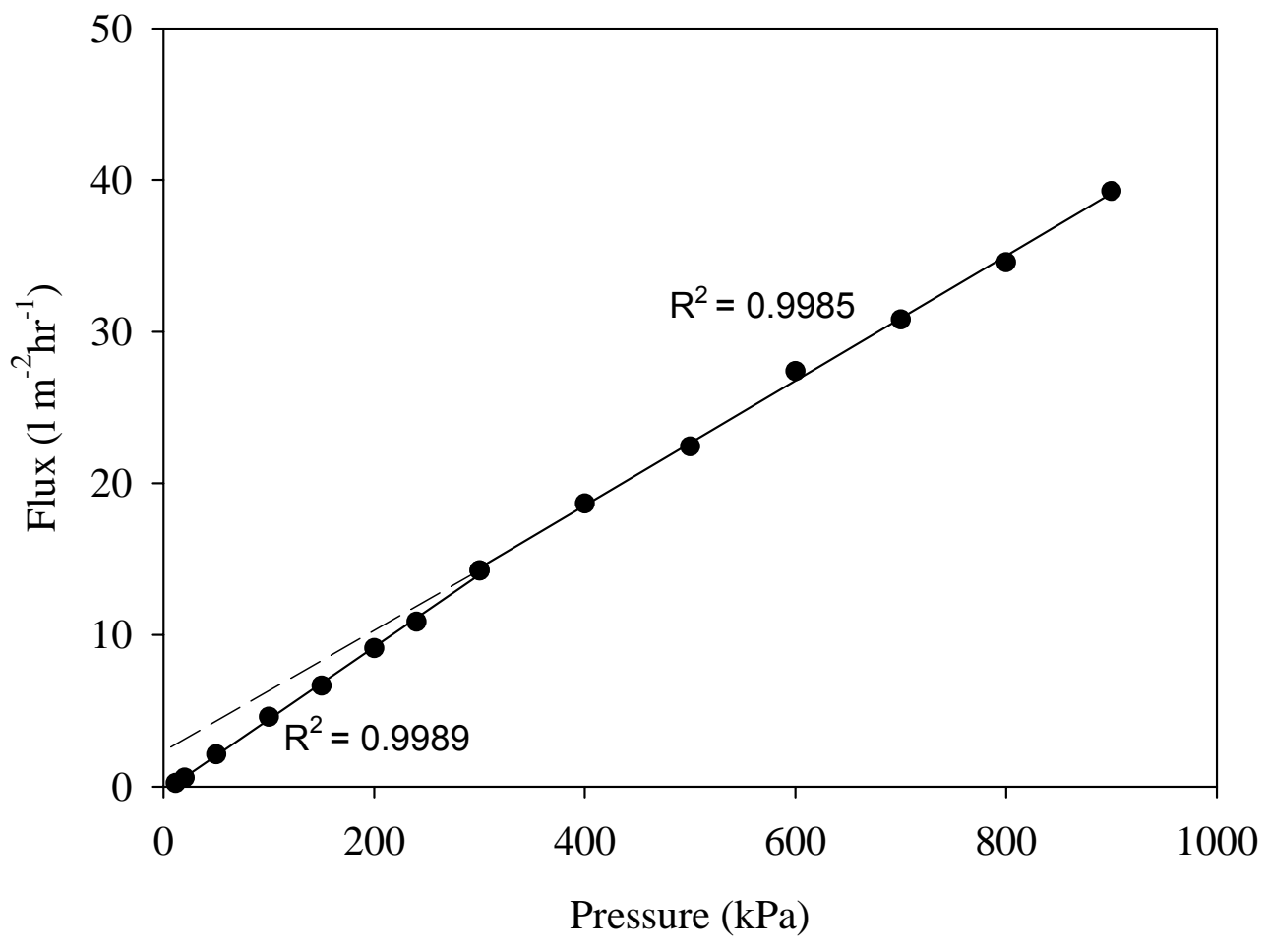

Figure 5 - Flux-pressure relationship for xylene at high and low pressures.

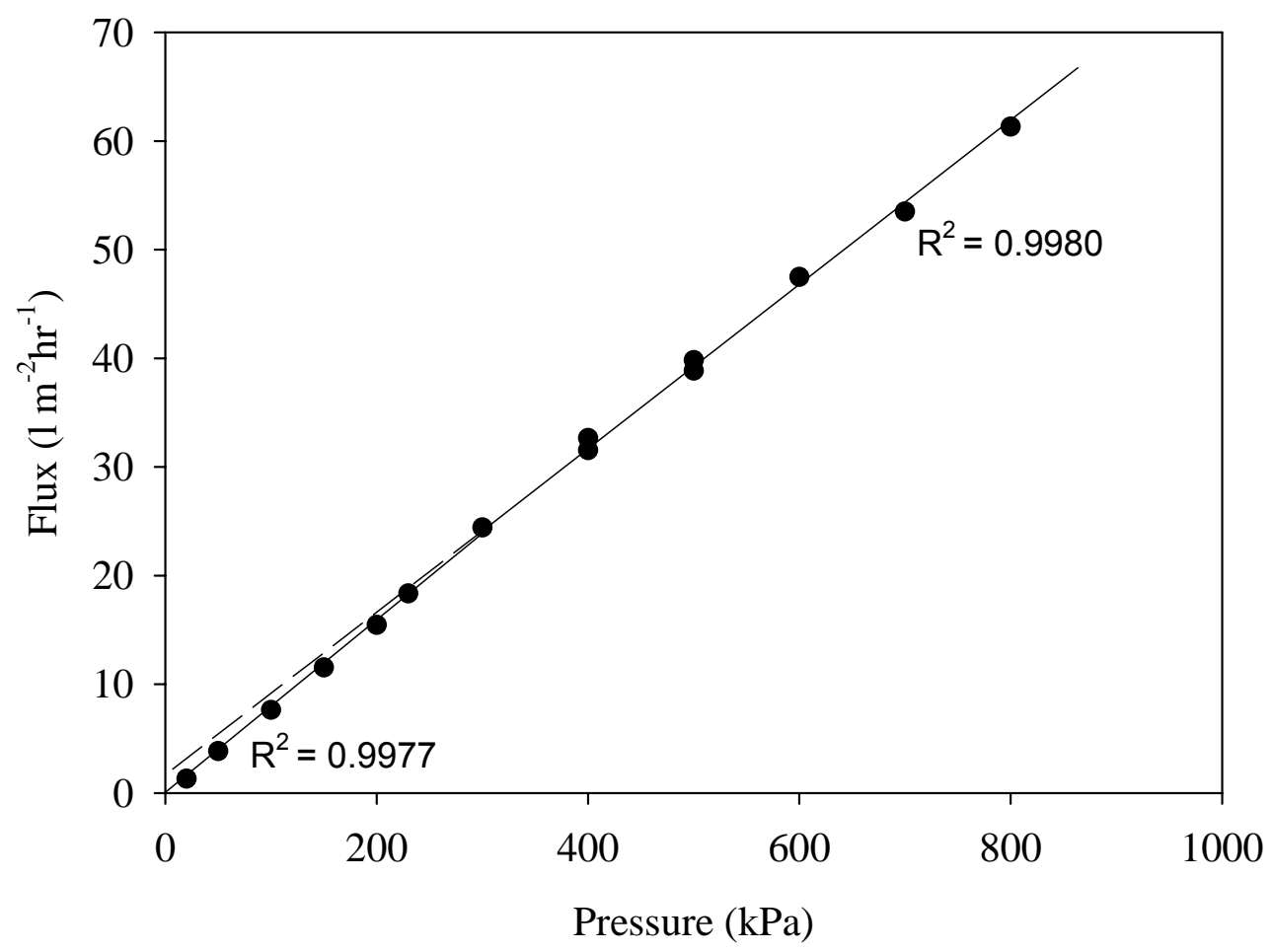

Figure 6 - Flux-pressure relationship for n-heptane at high and low pressures. 


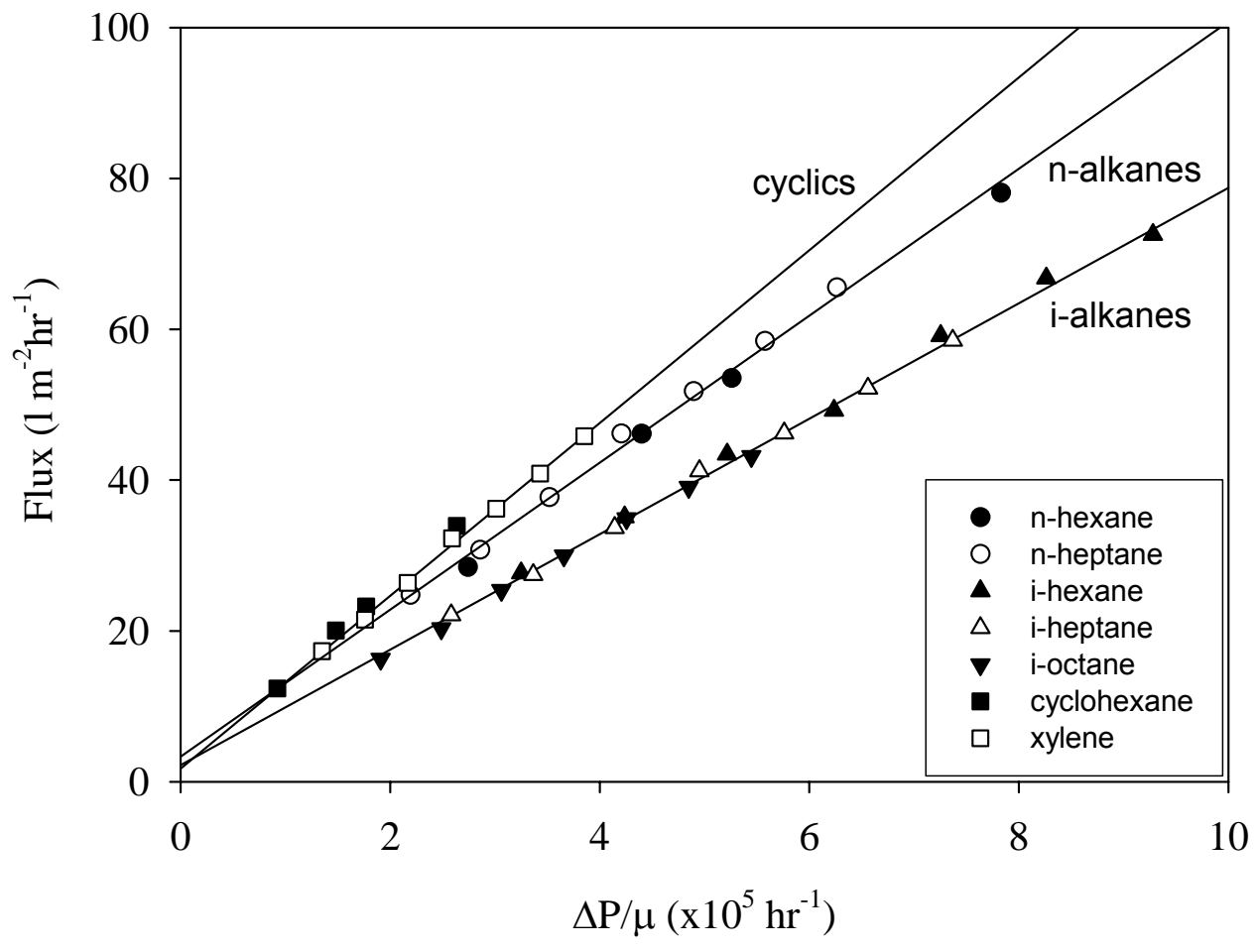

Figure 7 - Application of the Hagen-Poiseuille model for all solvents tested.

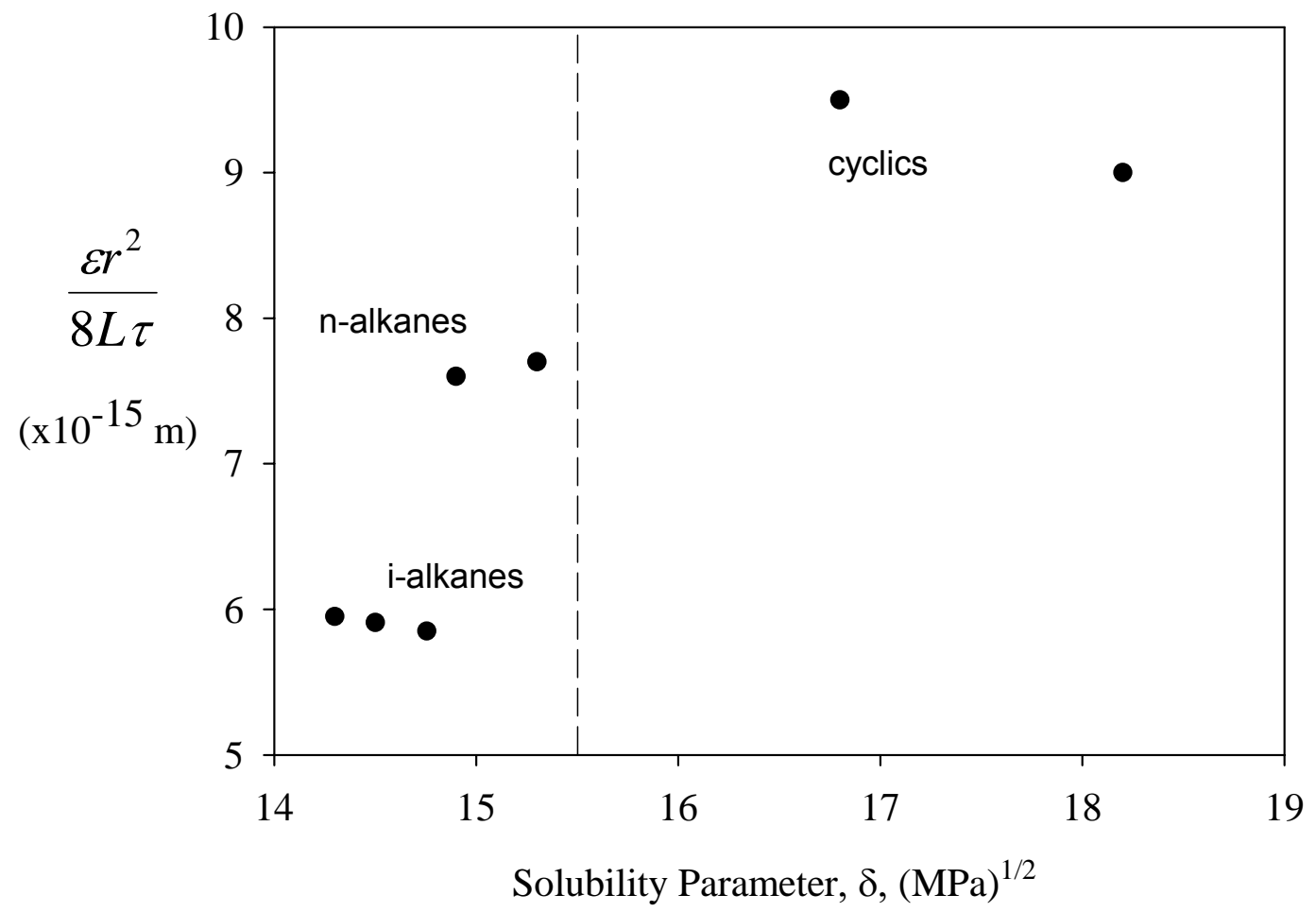

Figure 8 - Effect of swelling on the gradient of the Hagen-Poiseuille plot. The dotted line represents the solubility parameter of PDMS, $15.5 \mathrm{MPa}^{1 / 2}$. Values of $\delta$ are taken from [14]. 


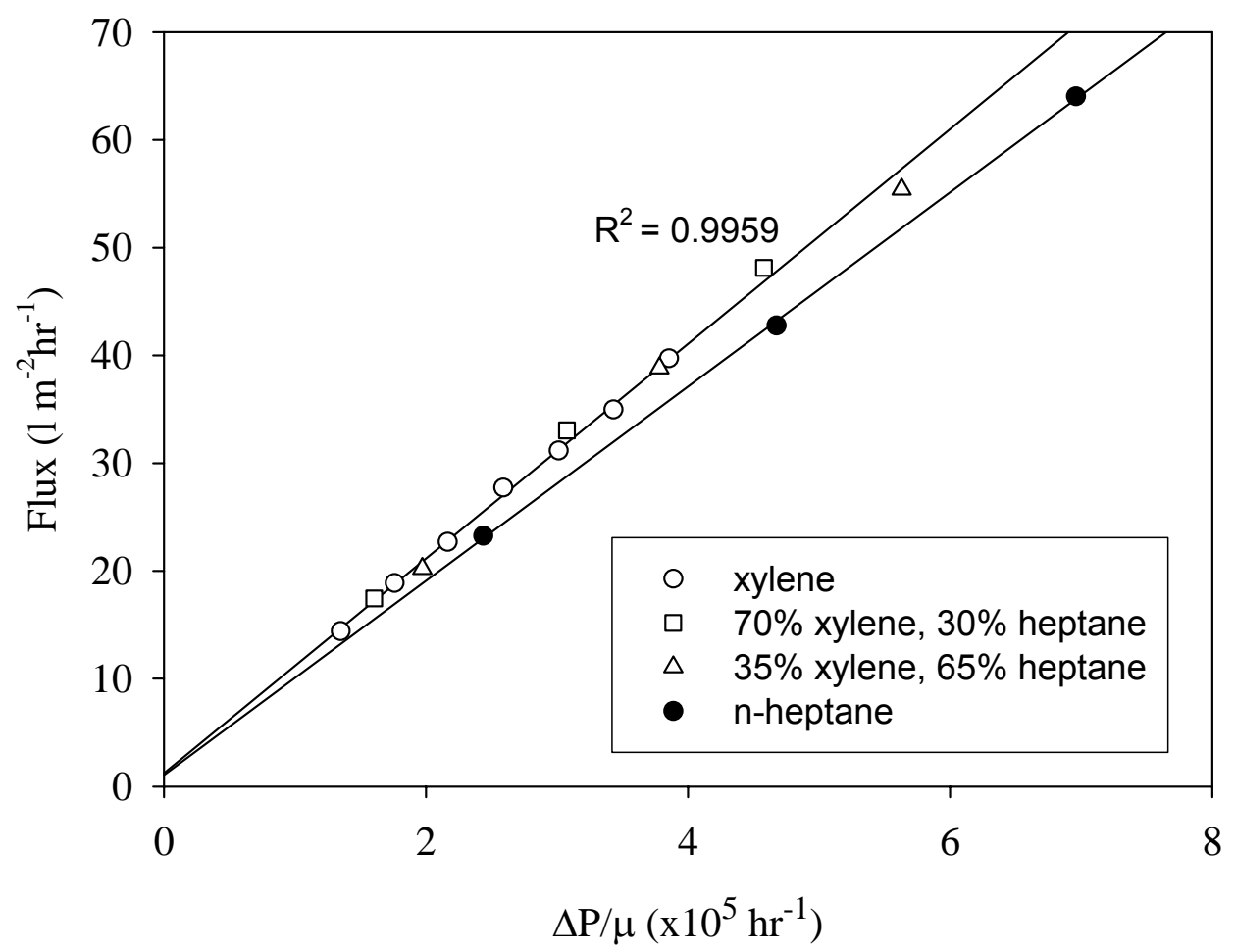

Figure 9 - Hagen-Poiseuille model for the xylene/n-heptane binary solvent system. $R^{2}$ value calculated with the n-heptane data omitted.

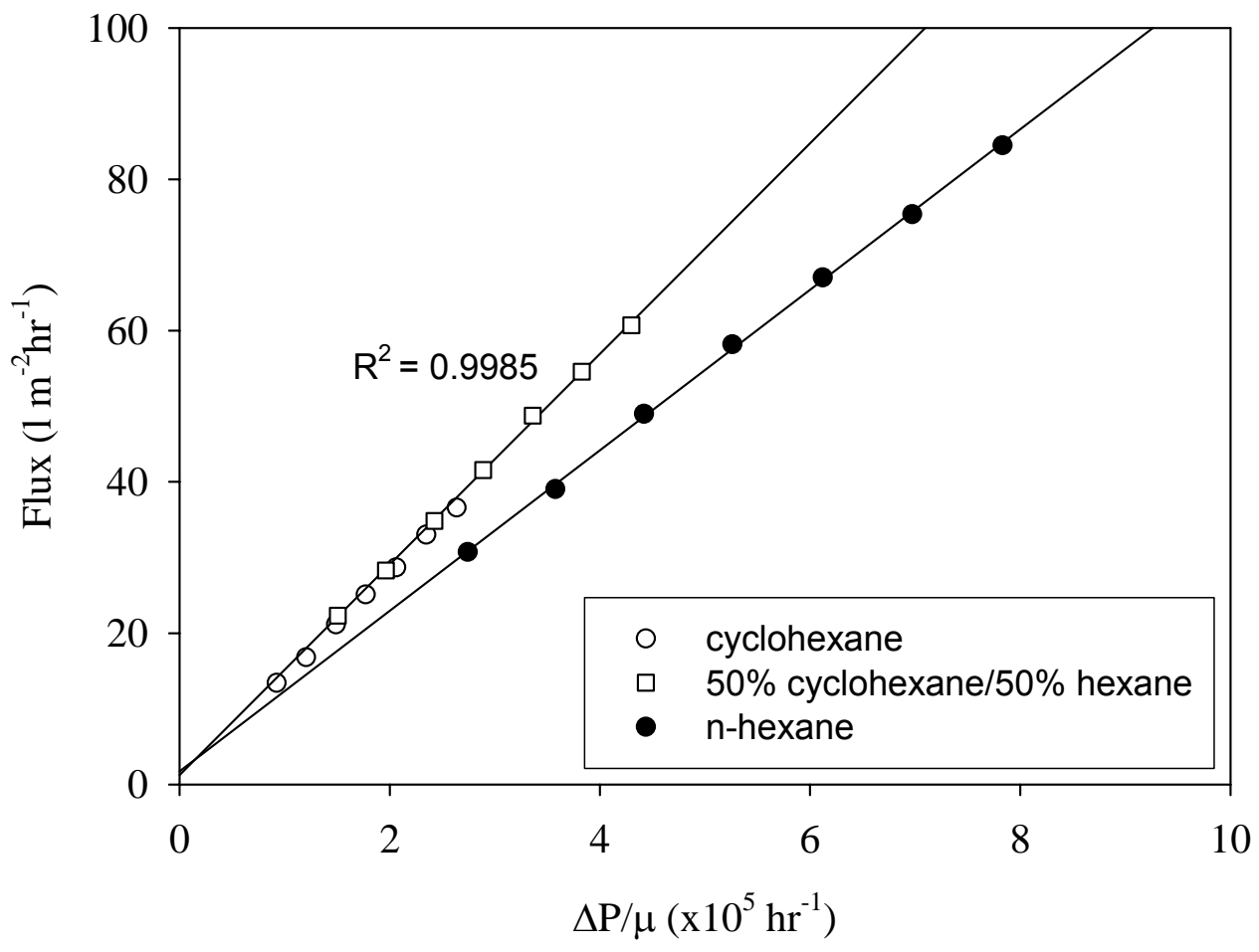

Figure 10 - Hagen-Poiseuille model for the $n$-hexane/cyclohexane binary solvent system. $R^{2}$ value calculated with the n-hexane data omitted. 


\begin{tabular}{|l|c|c|}
\hline Solvent & $\begin{array}{c}\text { Gradient } \\
\left(\times 10^{-2} \mathrm{I} \mathrm{m}^{-2} \mathrm{~h}^{-1} \mathrm{kPa}^{-1}\right)\end{array}$ & $\begin{array}{c}\text { Intercept } \\
\left(\mathrm{I} \mathrm{m}^{-2} \mathrm{~h}^{-1}\right)\end{array}$ \\
\hline n-hexane & 8.41 & 2.53 \\
n-heptane & 7.00 & 2.49 \\
i-hexane & 7.80 & 3.17 \\
i-heptane & 6.25 & 2.23 \\
i-octane & 4.66 & 1.56 \\
cyclohexane & 3.66 & 1.10 \\
xylene & 4.90 & 1.74 \\
\hline
\end{tabular}

Table 1 - Gradients and intercepts of the flux-pressure relationship in the $300-900 \mathrm{kPa}$ range.

\begin{tabular}{|l|c|c|c|}
\hline Classification & Solvent & $\begin{array}{c}\text { Density } \\
\left(\mathrm{kg} \mathrm{m}^{-3}\right)\end{array}$ & $\begin{array}{c}\text { Viscosity } \\
\left(\times 10^{-3} \mathrm{~Pa} \mathrm{~s}\right)\end{array}$ \\
\hline Straight-chain alkane & n-hexane & 660 & 0.32 \\
Branched alkane & n-heptane & 681 & 0.40 \\
& i-hexane & 653 & 0.27 \\
& i-heptane & 678 & 0.34 \\
Cyclic alkane & i-octane & 692 & 0.46 \\
Cyclic aromatic & cyclohexane & 779 & 0.95 \\
\hline
\end{tabular}

Table 2 - Solvent physical properties (taken from published data and estimations [21] at $20^{\circ} \mathrm{C}$ ). 